МИНИСТЕРСТВО ОБРАЗОВАНИЯ И НАУКИ РОССИЙСКОЙ ФЕДЕРАЦИИ

ТОМСКИЙ ГОСУДАРСТВЕННЫЙ УНИВЕРСИТЕТ

\title{
МАТЕРИАЛЫ
}

VIII Международной молодежной научной конференции

«МАТЕМАТИЧЕСКОЕ

И ПРОГРАММНОЕ ОБЕСПЕЧЕНИЕ

ИНФОРМАЦИОННЫХ, ТЕХНИЧЕСКИХ

И ЭКОНОМИЧЕСКИХ СИСТЕМ»

Томск, 26-30 мая 2021 г.

Под общей редакиией И.С. Шмырина

Томск

Издательство Томского государственного университета 2021 


\section{НЕЧЁТКИЙ АНАЛИЗ ФОРМАЛЬНЫХ ПОНЯТИЙ: ОБЗОР ПОДХОДОВ НА ПРИМЕРЕ}

Голышев В.К.

Сибирский федеральный университет

valeriygolyshev@mail.ru

\section{Введение}

Классический анализ формальных понятий работает с бинарными контекстами в виде $(0,1)$-матриц. На практике при измерении свойств объектов возникают ситуации, при которых оценка истинности утверждения «объект g обладает признаком т» является расплывчатой. Для оценивания таких суждений привлекают математический аппарат различных теорий неопределённости, таких, как теория нечётких множеств, грубых множеств, теории вероятностей или их комбинаций $[1,2]$. Внедрение нечёткой логики $[3,4,5]$ в классический анализ формальных понятий привело к возникновению и активному развитию нового направления интеллектуального анализа данных - нечёткий анализ формальных понятий (сокр. НАФП).

\section{1. Нечёткий анализ формальных понятий}

В нечётком анализе формальных понятий можно выделить восемь основных подходов [1,2], различающихся по

- структуре степеней истинности и сопряжённым операциям,

- способам определения нечёткого формального контекста,

- способам обобщения соответствий Галуа,

- способам определения нечёткого формального понятия.

Сравнение подходов по выделенным различиям представлено в табл. 1 и 2 (значок волны над множеством обозначает его нечёткость).

Таблица 1

Сравнение подходов по структурам степеней истинности и способу задания нечёткого контекста

\begin{tabular}{|c|c|c|c|c|}
\hline № & Подход & Автор, год & Контекст & Структура степеней истинности \\
\hline 1 & Решётка нечётких понятий & $\begin{array}{l}\text { Burusco и Fuentes- } \\
\text { Gonzalez, } 1994 \\
\text { Pollandt, } 1997 \\
\text { Belohlavek, } 1998\end{array}$ & $(G, M, \tilde{I})$ & Полная резидуальная решётка \\
\hline 2 & $\begin{array}{c}\text { Решётка нечётких понятий с } \\
\text { некоммутативной конъюнк- } \\
\text { цией }\end{array}$ & Georgescu 2002, 2003 & $(G, M, \tilde{I})$ & Обобщённая резидуальная решётка \\
\hline 3 & $\begin{array}{c}\text { Односторонняя решётка по- } \\
\text { нятий }\end{array}$ & $\begin{array}{c}\text { Yahiа и Јaoua, 2001, } \\
\text { Krajci, } 2003\end{array}$ & $(G, M, \tilde{I})$ & $\begin{array}{c}\text { Частично упорядоченное множество, } \\
\text { полная решётка, полная резидуальная } \\
\text { решётка } \\
\end{array}$ \\
\hline 4 & $\begin{array}{c}\text { Решётка чётко сгенерирован- } \\
\text { ных понятий }\end{array}$ & Belohlavek, 2005 & $(G, M, \tilde{I})$ & Полная резидуальная решётка \\
\hline 5 & $\begin{array}{c}\text { Обобщённая решётка поня- } \\
\text { тий }\end{array}$ & Krajci, 2005 & $(G, M, \tilde{I}, \otimes)$ & $\begin{array}{c}\text { Две полные решётки для объектов и } \\
\text { признаков, частично упорядоченное } \\
\text { множество степеней истинности с конъ- } \\
\text { юнкцией } \otimes\end{array}$ \\
\hline 6 & Мультисопряжённая решётка & Medina, 2007, 2009 & $(G, M, \tilde{I}, \sigma)$ & Мультисопряжённый фрейм \\
\hline 7 & Решётка с порогами & $\begin{array}{l}\text { Elloumi, 2004, } \\
\text { Zhang, } 2007\end{array}$ & $(G, M, \tilde{I})$ & Полная резидуальная решётка \\
\hline 8 & Решётка с замыканиями & $\begin{array}{c}\text { Belohlavek и } \\
\text { Vychodil, 2005, 2007, } \\
2012 \\
\end{array}$ & $(G, M, \tilde{I})$ & Полная резидуальная решётка \\
\hline
\end{tabular}


Сравнение подходов по способу обобщения соответствий Галуа и способу определения нечёткого формального понятия

\begin{tabular}{|c|c|c|c|}
\hline № & Подход & Стрелочные операторы (соответствия Галуа) & $\begin{array}{c}\text { Нечёткое формальное } \\
\text { понятие }\end{array}$ \\
\hline 1 & Нечёткая решётка понятий & $\begin{array}{l}\tilde{A}^{\uparrow}(m)=\bigwedge_{g \in G}(\tilde{A}(g) \rightarrow \tilde{I}(g, m)), \\
\tilde{B}^{\downarrow}(g)=\bigwedge_{m \in M}(\tilde{B}(m) \rightarrow \tilde{I}(g, m))\end{array}$ & $\begin{array}{l}(\tilde{A}, \tilde{B}): \\
\tilde{A}^{\uparrow}=\tilde{B} \\
\tilde{B}^{\downarrow}=\tilde{A}\end{array}$ \\
\hline 2 & $\begin{array}{l}\text { Решётка нечётких понятий } \\
\text { с некоммутативной конъ- } \\
\text { юнкцией }\end{array}$ & $\begin{array}{l}\tilde{A}^{\uparrow}(y)=\bigwedge_{g \in G}(\tilde{A}(g) \rightarrow \tilde{I}(g, m)), \\
\tilde{A}^{\Uparrow}(y)=\bigwedge_{g \in G}(\tilde{A}(g) \Rightarrow \tilde{I}(g, m)), \\
\tilde{B}^{\downarrow}(y)=\bigwedge_{m \in M}(\tilde{B}(m) \rightarrow \tilde{I}(g, m)), \\
\tilde{B}^{\Downarrow}(y)=\bigwedge_{m \in M}(\tilde{B}(m) \Rightarrow \tilde{I}(g, m))\end{array}$ & $\begin{array}{c}\left(\tilde{A}, \tilde{B}_{1}, \tilde{B}_{2}\right): \\
\tilde{A}^{\uparrow}=\tilde{B}_{1}, \\
\tilde{A}^{\Uparrow}=\tilde{B}_{2}, \\
\tilde{B}_{1}^{\Downarrow}=\tilde{A}, \\
\tilde{B}_{2}^{\downarrow}=\tilde{A}\end{array}$ \\
\hline 3 & $\begin{array}{l}\text { Односторонняя решётка } \\
\text { понятий }\end{array}$ & $\begin{array}{c}f(A)(m)=\bigwedge_{g \in A} \tilde{I}(g, m) \\
h(\tilde{B})=\{g \in G \mid \forall m \in M: \tilde{B}(m) \leq \tilde{I}(g, m)\}\end{array}$ & $\begin{array}{l}(A, \tilde{B}): \\
f(A)=\tilde{B} \\
h(\tilde{B})=A\end{array}$ \\
\hline 4 & $\begin{array}{l}\text { Решётка чётко сгенериро- } \\
\text { ванных понятий }\end{array}$ & $\begin{array}{l}\tilde{A}^{\uparrow}(m)=\bigwedge_{g \in G}(\tilde{A}(g) \rightarrow \tilde{I}(g, m)), \\
\tilde{B}^{\downarrow}(g)=\bigwedge_{m \in M}(\tilde{B}(m) \rightarrow \tilde{I}(g, m)) .\end{array}$ & $\begin{array}{c}(\tilde{A}, B): \\
\exists B_{c} \subseteq M \\
\text { такое, что } \\
\tilde{A}=B_{c}^{\downarrow} \text { и } B=B_{c}^{\downarrow \uparrow}\end{array}$ \\
\hline 5 & $\begin{array}{l}\text { Обобщённая решётка поня- } \\
\text { тий }\end{array}$ & $\begin{array}{l}\tilde{A}^{\uparrow}(m)=\sup \left\{b \in L_{M} \mid \forall g \in G: \tilde{A}(g) \otimes b \leq \tilde{I}(g, m)\right\}, \\
\tilde{B}^{\downarrow}(g)=\sup \left\{a \in L_{G} \mid \forall m \in M: a \otimes \tilde{B}(m) \leq \tilde{I}(g, m)\right\}\end{array}$ & $\begin{array}{l}(\tilde{A}, \tilde{B}): \\
\tilde{A}^{\uparrow}=\tilde{B} \\
\tilde{B}^{\downarrow}=\tilde{A}\end{array}$ \\
\hline 6 & $\begin{array}{l}\text { Мультисопряжённая ре- } \\
\text { шётка }\end{array}$ & $\begin{array}{l}\tilde{A}^{\uparrow_{\sigma}}(g)=\inf \left\{\tilde{I}(g, m) \square^{\sigma(m)} \tilde{A}(m) \mid m \in M\right\}, \\
\tilde{B}^{\downarrow_{\sigma}}(m)=\inf \left\{\tilde{I}(g, m) \square_{\sigma(g)} \tilde{B}(g) \mid g \in G\right\}\end{array}$ & $\begin{array}{l}(\tilde{A}, \tilde{B}): \\
\tilde{A}^{\uparrow_{\sigma}}=\tilde{B} \\
\tilde{B}^{\downarrow_{\sigma}}=\tilde{A}\end{array}$ \\
\hline \multirow{3}{*}{7} & \multirow{3}{*}{ Решётка с порогами } & $\begin{aligned} C^{*} & =\left\{m \in M \mid \inf _{g \in G}(C(g) \rightarrow \tilde{I}(g, m)) \geq \delta\right\}, \\
D^{*} & =\left\{g \in G \mid \inf _{m \in M}(D(m) \rightarrow \tilde{I}(g, m)) \geq \delta\right\}\end{aligned}$ & $\begin{array}{l}(C, D): \\
C^{*}=D \\
D^{*}=C\end{array}$ \\
\hline & & $\begin{array}{c}C^{\square}(m)=\left(\delta \rightarrow \inf _{g \in C} \tilde{I}(g, m)\right), \\
\tilde{B}^{\square}=\left\{g \in G \mid \inf _{m \in M}(\tilde{B}(m) \rightarrow \tilde{I}(g, m)) \geq \delta\right\}\end{array}$ & $\begin{array}{l}(C, \tilde{B}): \\
C^{\square}=\tilde{B}, \\
\tilde{B}^{\square}=C\end{array}$ \\
\hline & & $\begin{array}{c}\tilde{A}^{\square}=\left\{m \in M \mid \inf _{g \in G}(\tilde{A}(g) \rightarrow \tilde{I}(g, m)) \geq \delta\right\}, \\
D^{\square}(g)=\left(\delta \rightarrow \inf _{m \in D} \tilde{I}(g, m)\right)\end{array}$ & $\begin{array}{l}(\tilde{A}, D): \\
\tilde{A}^{\square}=D, \\
D^{\square}=\tilde{A} .\end{array}$ \\
\hline 8 & Решётка с замыканиями & $\begin{array}{l}\tilde{A}^{\uparrow}(m)=\inf _{g \in G}\left(\tilde{A}(g)^{*_{g}} \rightarrow \tilde{I}(g, m)\right), \\
\tilde{B}^{\downarrow}(g)=\inf _{m \in M}\left(\tilde{B}(m)^{*_{m}} \rightarrow \tilde{I}(g, m)\right)\end{array}$ & $\begin{array}{l}(\tilde{A}, \tilde{B}): \\
\tilde{A}^{\uparrow}=\tilde{B} \\
\tilde{B}^{\downarrow}=\tilde{A}\end{array}$ \\
\hline
\end{tabular}

Основополагающим подходом стал подход №1, положивший начало активному развитию НАФП [6,7]. Подход №2 имеет место в ситуациях, когда важен порядок между членами конъюнкции, т.к. опускает требование её коммутативности [8]. Односторонние решётки $[9,10]$ содержат нечёткие понятия, состоящие из объёмов, представленных чёткими множествами, и из содержаний в виде нечётких множеств. Подход №4 использует определение чётко сгенерированного понятия [11]. Полученная решётка изоморфна односторонней решётке с нечёткими объемами и чёткими содержаниями. 
Обобщённые решётки понятий $[12,13]$ включают в себя некоторые другие подходы (подход №1, №3, №8). Мультисопряжённые решётки используют так называемые бирезидуальные структуры $[14,15]$ и включают в себя подход №1, а также обобщённые решётки понятий. Решётки с порогами $[16,17,18]$ используют параметр, являющийся некоторой степенью истинности и использующийся для контроля числа извлекаемых понятий. Решётки с замыканиями $[2,5,19]$ используют функцию истинности унарной логической связки «очень», также позволяющую контролировать число получаемых нечётких понятий. Включает в себя подход №7 с порогами.

\section{2. Демонстрация работы соответствий Галуа в различных подходах на примере}

Для примера был взят нечёткий формальный контекст с тремя объектами $\{1,2,3\}$ и тремя признаками $\{a, b, c\}$ (табл. 3).

Пример нечёткого формального контекста

\begin{tabular}{|r|r|r|l|}
\hline & \multicolumn{1}{|c|}{$a$} & \multicolumn{1}{c|}{$b$} & \multicolumn{1}{c|}{$c$} \\
\hline 1 & 1 & 0 & 0.3 \\
\hline 2 & 0 & 0.8 & 0.5 \\
\hline 3 & 0.6 & 0.1 & 0.5 \\
\hline
\end{tabular}

В качестве сопряжённых операций рассматривались нечёткая конъюнкция и импликация Лукасевича:

$$
a \otimes b=\max \{0, a+b-1\}, a \rightarrow b=\min \{1,1-a+b\} .
$$

Для множества объектов были взяты нечёткое множество $\left\{\left.2\right|_{0.5},\left.3\right|_{0.1}\right\}$ и множество $\{2,3\}$ в зависимости от требований подхода. С помощью обобщений Галуа находились множества признаков (табл. 4, столбец 4), после чего данные множества отображались обратно во множества объектов (табл. 4, столбец 5). В столбце 6 табл. 4 указывается, составляет ли полученная пара множеств объектов и признаков нечёткое формальное понятие в смысле рассматриваемого подхода.

Таблица 4

\begin{tabular}{|c|c|c|c|c|c|}
\hline \multicolumn{6}{|c|}{ Примеры обобщённых соответствий Галуа в различных подходах НАФП } \\
\hline № & Подход & $\tilde{A}$ & $\tilde{\boldsymbol{B}}=\tilde{\boldsymbol{A}}^{\uparrow}$ & $\tilde{\boldsymbol{B}}^{\downarrow}=\tilde{\boldsymbol{A}}^{\uparrow \downarrow}$ & $\begin{array}{c}(\tilde{A}, \tilde{B})- \\
\text { нечёткое понятие }\end{array}$ \\
\hline 1 & Решётка нечётких понятий & $\left\{\left.2\right|_{0.5},\left.3\right|_{0.1}\right\}$ & $\left\{\left.a\right|_{0.5}, b, c\right\}$ & $\left\{\left.2\right|_{0.5},\left.3\right|_{0.1}\right\}$ & да \\
\hline 2 & $\begin{array}{l}\text { Решётка нечётких понятий } \\
\text { с некоммутативной конъ- } \\
\text { юнкцией }\end{array}$ & $\left\{\left.2\right|_{0.5},\left.3\right|_{0.1}\right\}$ & $\begin{array}{l}\tilde{B}_{1}=\tilde{A}^{\uparrow}=\left\{\left.a\right|_{0.5}, b, c\right\} \\
\tilde{B}_{2}=\tilde{A}^{\Uparrow}=\left\{\left.a\right|_{0.5}, b, c\right\}\end{array}$ & $\begin{aligned} \tilde{B}_{1}^{\Downarrow} & =\left\{\left.2\right|_{0.5},\left.3\right|_{0.1}\right\}, \\
\tilde{B}_{2}^{\downarrow} & =\left\{\left.2\right|_{0.5},\left.3\right|_{0.1}\right\}\end{aligned}$ & да \\
\hline 3 & $\begin{array}{l}\text { Односторонняя решётка } \\
\text { понятий }\end{array}$ & $\{2,3\}$ & $\left\{\left.b\right|_{0.1},\left.c\right|_{0.5}\right\}$ & $\{2,3\}$ & да \\
\hline 4 & $\begin{array}{l}\text { Решётка чётко сгенериро- } \\
\text { ванных понятий }\end{array}$ & $\left\{\left.2\right|_{0.5},\left.3\right|_{0.1}\right\}$ & $\left\{\left.a\right|_{0.5}, b, c\right\}$ & $\left\{\left.2\right|_{0.5},\left.3\right|_{0.1}\right\}$ & нет \\
\hline 5 & $\begin{array}{l}\text { Обобщённая решётка } \\
\text { понятий }\end{array}$ & $\left\{\left.2\right|_{0.5},\left.3\right|_{0.1}\right\}$ & $\{b, c\}$ & $\left\{\left.2\right|_{0.5},\left.3\right|_{0.1}\right\}$ & да \\
\hline 6 & $\begin{array}{l}\text { Мультисопряжённая ре- } \\
\text { шётка понятий } \\
\end{array}$ & $\left\{\left.2\right|_{0.5},\left.3\right|_{0.1}\right\}$ & $\{b, c\}$ & $\left\{\left.2\right|_{0.5}\right\}$ & нет \\
\hline \multirow{3}{*}{7} & \multirow{3}{*}{$\begin{array}{l}\text { Решётка с порогами, } \\
\delta=0.3\end{array}$} & $\{2,3\}$ & $D=C^{*}=\{c\}$ & $D^{*}=\{1,2,3\}$ & нет \\
\hline & & $\{2,3\}$ & $\tilde{B}=C^{\square}=\left\{\left.a\right|_{0.7},\left.b\right|_{0.8}, c\right\}$ & $\tilde{B}^{\square}=\{2,3\}$ & да \\
\hline & & $\left\{\left.2\right|_{0.5},\left.3\right|_{0.1}\right\}$ & $D=\tilde{A}^{\square}=\{a, b, c\}$ & $D^{\square}=\left\{\left.1\right|_{0.7},\left.2\right|_{0.7},\left.3\right|_{0.8}\right\}$ & нет \\
\hline \multirow{2}{*}{8} & \multirow{2}{*}{ Решётка с замыканиями } & $\left\{\left.2\right|_{0.5},\left.3\right|_{0.1}\right\}$ & $\begin{array}{c}\text { идентичность } \\
\tilde{B}=\tilde{A}^{\uparrow}=\left\{\left.a\right|_{0.5}, b, c\right\}\end{array}$ & 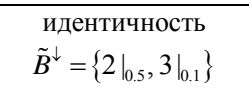 & да \\
\hline & & $\left\{\left.2\right|_{0.5},\left.3\right|_{0.1}\right\}$ & $\begin{array}{c}\text { глобализация } \\
\tilde{B}=\tilde{A}^{\uparrow}=\{a, b, c\}\end{array}$ & $\begin{array}{c}\text { глобализация } \\
\tilde{B}^{\downarrow}=\left\{\left.3\right|_{0.1}\right\}\end{array}$ & нет \\
\hline
\end{tabular}


Продемонстрируем процесс нахождения обобщённых соответствий Галуа в подходе №3, предполагая, что множество объектов $A=\{2,3\}$ - чёткое (табл. 4, подход №3). Результат отображения $f(A)$ есть нечёткое множество объектов $\tilde{B}=f(A)=\left\{\left.2\right|_{0.1},\left.3\right|_{0.5}\right\}$ Действительно,

$$
\begin{aligned}
& f(A)(a)=\wedge_{g \in A} \tilde{I}(g, a)=\tilde{I}(2, a) \wedge \tilde{I}(3, a)=0 \wedge 0.6=0, \\
& f(A)(b)=\bigwedge_{g \in A} \tilde{I}(g, b)=\tilde{I}(2, b) \wedge \tilde{I}(3, b)=0.8 \wedge 0.1=0.1, \\
& f(A)(c)=\bigwedge_{g \in A} \tilde{I}(g, c)=\tilde{I}(2, c) \wedge \tilde{I}(3, c)=0.5 \wedge 0.5=0.5 .
\end{aligned}
$$

Далее вычислим $h(\tilde{B})$ :

$$
\begin{aligned}
& g=1: \tilde{B}(a) \leq \tilde{I}(1, a), 0 \leq 1, \\
& \tilde{B}(b) \leq \tilde{I}(1, b), 0.1>0, \\
& g=2: \tilde{B}(a) \leq \tilde{I}(2, a), 0 \leq 0, \\
& \tilde{B}(b) \leq \tilde{I}(2, b), 0.1 \leq 0.8 \\
& \tilde{B}(c) \leq \tilde{I}(2, c), 0.5 \leq 0.5, \\
& g=3: \tilde{B}(a) \leq \tilde{I}(3, a), 0 \leq 0.6, \\
& \tilde{B}(b) \leq \tilde{I}(3, b), 0.1 \leq 0.1, \\
& \tilde{B}(c) \leq \tilde{I}(3, c), 0.5 \leq 0.5 .
\end{aligned}
$$

Значит, множество $h(\tilde{B})=\{2,3\}$. Поскольку $A=h(\tilde{B})$, пара множеств $(A, \tilde{B})$ является нечётким формальным понятием в смысле подхода №3.

\section{Заключение}

В данной работе произведен обзор подходов НАФП, для них приведены сравнительные таблицы. Для демонстрации работы обобщённых соответствий Галуа представлен простой пример на небольшом нечётком контексте, результаты вычислений собраны в таблицу, показывающую различия работы стрелочных операторов. Кроме того, для подходов №1, №8 реализованы алгоритмы, позволяющие находить все нечёткие формальные понятия заданного нечёткого контекста. Однако применение НАФП ограничено в связи с высокой вычислительной сложностью, что говорит об актуальности исследований по разработке эффективных алгоритмов решения задачи нахождения всех нечётких формальных понятий.

\section{ЛИТЕРАТУРА}

1. Poelmans J., Ignatov D.I., Kuznetsov S.O., Dedene G. Fuzzy and rough formal concept analysis: a survey // International Journal of General Systems. - 2014. - P. 105-134.

2. Belohlavek R., Vychodil V. What is a fuzzy concept lattice? // Proc. CLA 2005. - 3rd Int. Conference on Concept Lattices and Their Applications. - 162. - P. 34-45.

3. Zadeh L.A. Fuzzy sets // Information and control. - 1965. - 8. - P. 338-353.

4. Новак В., Перфильева И., Мочкорж И. Математические принципы нечеткой логики // Пер. с англ.; под ред. Аверкина А.Н. - М.: ФИЗМАТЛИТ, 2006. - 352 с.

5. Гольшев В.К. Методы нечёткой логики в анализе формальных понятий // Математическое и программное обеспечение информационных, технических и экономических систем: Материалы VII Международной молодежной научной конференции. - Томск, 2019. - С. 269-276.

6. Belohlavek R. Fuzzy Concepts and Conceptual Structures: Induced Similarities // Joint Conf. Inf. Sci. 1998, Durham, NC. - 1998. - V.I. - P. 179-182.

7. Belohlavek R. Fuzzy Galois Connections // Mathematical Logic Quarterly - 1995. 45. - P. 497-504.

8. Georgescu G., Popescu A. Concept Lattices and Similarity in Non-Commutative Fuzzy Logic // Fund. Inform. 2002.- 55 (1). - P. 23-54. 
9. Yahia S.B., Jaoua A. Discovering Knowledge from Fuzzy Concept Lattice // Data Mining and Computational Intelligence. Studies in Fuzziness and Soft Computing. - Physica, Heidelberg. - 2001. - V. 68. - P. 167-190. 530.

10. Krajci S. Cluster based efficient generation of fuzzy concepts // Neural Network World. - 2003.- 13. - P. 521-

11. Belohlavek R., Sklenar V., Zacpal J. Crisply Generated Fuzzy Concepts // B. Ganter et al. eds. ICFCA, LNAI. $-2005-3403$ - P. 269-284.

12. Krajci S. A generalized concept lattice // Logic J. IGPL. - 2005. - 13(5). - P. 543-550.

13. Medina J., Ojeda-Aciego M., Ruiz-Calvino J. Relating Generalized Concept Lattices and Concept Lattices for Non-Commutative Conjunctors // Applied Mathematics Letters. - 2008. - 2. - P. 1296-1300.

14. Medina J., Ojeda-Aciego M., Ruiz-Calvino J. On Multi-adjoint Concept Lattices: Definition and Representation Theorem // ICFCA. LNAI. - 2007. - 4390. - P. 197-209.

15. Medina J., Ojeda-Aciego M., Ruiz-Calvino J. Formal Concept Analysis via Multi-Adjoint Concept Lattices // Fuzzy Sets and Systems. - 2009. - 160. - P. 130-144.

16. Elloumi S., Jaam J., Hasnah A., Jaoua A., Nafkha I. A Multi-Level Conceptual Data Reduction Approach Based on the Lukasiewicz Implication // Information Sciences. - 2004. - 163. - P. 253-262.

17. Shao M.W., Zhang W.X. Information Granularity Lattices // Proc. of the Sixth IEEE Int. Conf. on Machine Learning and Cybernetics. - Hong Kong, 2007.

18. Belohlavek $R$. A note on variable threshold concept lattices: Threshold-based operators are reducible to classical concept-forming operators // Information Sciences. - 2007. - 177. - P. 3186-3191.

19. Belohlavek R., Vychodil V. Fuzzy concept lattices constrained by hedges // Journal of Advanced Computational Intelligence and Intelligent Informatics. - 2007. - 11(6). - P. 536-545.

DOI: $10.17223 / 978-5-907442-42-9-2021-6$

\title{
ПРИМЕНЕНИЕ МЕТОДОВ ГЛУБОКОГО ОБУЧЕНИЯ К ЗАДАЧЕ КЛАССИФИКАЦИИ ВРЕМЕННЫХ РЯДОВ
}

\author{
Дуля И.С., Мурзагулов Д.А. \\ Томский государственный университет \\ idulya7@gmail.com, murzagulovdamir@gmail.com
}

\section{Введение}

В эпоху Индустрии 4.0 компании все больше сосредотачиваются на разработке технологичных решений и стремятся максимально перестроить существующие бизнеспроцессы под новую концепцию, основанную на сборе и анализе данных. Большая часть собираемых данных на предприятиях представлена в виде сигналов (данные, динамически изменяющиеся во времени). Например, электрические импульсы, данные с акселерометров, давление в устройствах и т.д. В целях компьютерной обработки исходные аналоговые сигналы дискретизируют и представляют в виде временных рядов. Далее к данным применяют статистические модели и методы машинного обучения, позволяющие извлечь ценные знания для бизнеса, которые используются в деятельности предприятия и оптимизации производства. В данном контексте классификация временных рядов была и остаётся одной из наиболее популярных задач интеллектуального анализа данных [1]

С ростом числа открытых наборов данных были предложены сотни подходов и алгоритмов решения задачи классификации временных рядов. Можно выделить три основные группы подходов к решению задачи классификации временных рядов. Первый подход самый очевидный: на вход статистической модели подаются значения временного ряда. Здесь существует ограничение на то, что временные ряды должны быть одинаковой длины, кроме того, малейшее смещение может привести к существенному искажению результата классификации. В основе второго подхода лежит использование метрических моделей (например, модель $k$-ближайших соседей) с «эластичной» метрикой расстояния. Третий подход предполагает переход из временного пространства в пространство признаков. В рамках подхода исследователь самостоятельно подбирает характеристики, полезные в решении конкретной задачи. Ключевая проблема состоит в том, что разные аналитики для одной задачи подберут разные наборы характеристик. Отсюда возникает проблема: невозможно определить является ли предложенный пере- 